\title{
Expression of SHP-1 Phosphatase Indicates Post-Germinal Center Cell Derivation of B-Cell Posttransplant Lymphoproliferative Disorders
}

\author{
Michele Paessler, Plamen Kossev, Donald Tsai, Puthiaveetil Raghunath, \\ Miroslaw Majewski, Qian Zhang, Preetha Ramalingam, Stephen Schuster, \\ John Tomaszewski, Daniel A. Arber, Eric Hsi, and Mariusz A. Wasik
}

Department of Pathology and Laboratory Medicine (MP, PK, P. Raghunath, MM, QZ, JT, MAW) and Division of Hematology and Oncology (DT, SS), University of Pennsylvania, Philadelphia, Pennsylvania; Cleveland Clinic Foundation (P. Ramalingam, EH), Cleveland, Ohio; and City of Hope Medical Center (DA), Duarte, California

\begin{abstract}
SUMMARY: SHP-1 tyrosine phosphatase acts as a negative regulator of signaling by receptors for growth factors, cytokines, and chemokines and by receptors involved in immune response. Our recent study showed that SHP-1 is tightly regulated at various stages of $\mathrm{B}$-cell differentiation and is expressed in the mantle and marginal zones, interfollicular $\mathrm{B}$ cells, and plasma cells, whereas it is nondetectable in germinal center cells. In this study we evaluated expression of SHP-1 in vitro and in vivo in nine cell lines representing three different types of $\mathrm{EBV}^{+} \mathrm{B}$-cell populations closely resembling or derived from posttransplant lymphoproliferative disorders (PTLDs). Furthermore, we examined tissue samples from 58 patients with B-cell PTLDs, both EBV ${ }^{+}$ ( $85 \%$ of the cases analyzed) and EBV ${ }^{-}(15 \%)$. SHP-1 protein was strongly expressed in all cell lines and PTLD cases. In addition, the PTLD cases were essentially negative for germinal center B-cell markers: none expressed CD10 and only one expressed BCL-6. More than 40\% expressed a late post-germinal B-cell marker, CD138. The universal expression of SHP-1, lack of expression of CD10 and BCL-6, and frequent expression of CD138 suggest that PTLDs are derived from post-germinal center $B$ cells regardless of the EBV cell infection status. Based on the immunophenotype, B-cell PTLDs could be divided into two broad categories corresponding to the early $\left(\mathrm{CD} 10^{-} / \mathrm{BCL}-6^{-} / \mathrm{SHP}-1^{+} / \mathrm{CD} 138^{-}\right)$and late $\left(\mathrm{CD} 10^{-} / \mathrm{BCL}^{-} 6^{-} / \mathrm{SHP}-1^{+} / \mathrm{CD} 138^{+}\right)$postgerminal center cells. By being expressed earlier, SHP-1 is a more sensitive marker of post-germinal center B cells than CD138, which is seen on the terminally differentiated immunoblasts and plasma cells. (Lab Invest 2002, 82:1599-1606).
\end{abstract}

$T$ he protein phosphotyrosine phosphatase SHP-1 plays an important role in modulating intracellular signaling events and in regulating cell activation, proliferation, differentiation, and migration (Zhang et al, 2000a). SHP-1 phosphatase is a negative regulator of signaling through receptors for cytokines, growth factors, and chemokines and through receptors involved in immune responses such as B-and T-antigen receptors (Haque et al, 1998; Zhang et al, 2000b). SHP-1 down-regulates cell activation by binding and dephosphorylating the receptors and receptorassociated tyrosine kinases (Haque et al, 1998; Wu et al, 1998; Zhang et al, 2000b).

We (Kossev et al, 2001) and others (Delibrias et al, 1997) have shown that SHP-1 expression is tightly regulated in normal $B$ cells at various stages of their maturation. Germinal center B cells lack SHP-1 ex-

DOI: 10.1097/01.LAB.0000036873.16297.A5

Received August 26, 2002.

This study was supported in part by Grant CA89194 from the National Cancer Institute (to MAW).

Address reprint requests to: Dr. Mariusz A. Wasik, 7.106 Founders Building, Department of Pathology and Laboratory Medicine, University of Pennsylvania Medical Center, 3400 Spruce Street, Philadelphia, Pennsylvania19104.E-mail:wasik@mail.med.upenn.edu pression, whereas the pre-germinal center mantle zone cells express the protein. The post-germinal center marginal zone cells, interfollicular B cells, and plasma cells are also positive for SHP-1 expression. Based on these data, we have analyzed SHP-1 expression in several types of small B-cell lymphomas: mantle and marginal zone lymphomas and small lymphocytic lymphoma/chronic lymphocytic lymphoma. The differential pattern of SHP-1 expression in these lymphomas closely resembled the pattern seen in their corresponding normal B-cell counterparts.

Posttransplant lymphoproliferative disorders (PTLDs) comprise a heterogeneous group of lymphoproliferative disorders that represent a major complication of induced immunosuppression for organ transplantation (Harris et al, 1997; Swierdlow, 1997). They encompass a spectrum of lymphoproliferations ranging from early reactive hyperplasias to overt lymphomas, usually of B-cell origin. The World Health Organization Classification (Harris et al, 2001) categorizes PTLDs into four broad, yet distinct, categories: (1) early lesions, such as plasmacytic hyperplasias and infectious mononucleosis-like lesions, which are usually polyclonal; (2) polymorphic PTLDs, which almost always show clonal immunoglobulin gene rearrangements by PCR-based evaluation; (3) monomorphic PTLDs, including B-cell neoplasms such as diffuse large 
B-cell lymphoma, Burkitt/Burkitt-like lymphoma, and plasma cell myeloma, which virtually always show clonal immunoglobulin gene rearrangements and frequently display structural genetic abnormalities; and (4) Hodgkin/Hodgkin-like PTLD. The pathogenesis of PTLDs is not fully understood; however, EBV, a gammaherpesvirus that preferentially infects B lymphocytes, has long been implicated in their development (Tanner and Alfieri, 2001; Tao and Wasik, 2001). Induction of an activated, differentiated B-cell phenotype is one of EBV effects on the infected $B$ cells.

Because SHP-1 is expressed by post-germinal center, but not germinal center, cells (Delibrias et al, 1997; Kossev et al, 2001), we analyzed PTLD-related EBV ${ }^{+}$ B-cell lines and PTLD tissues for expression of SHP-1 protein to characterize the histogenesis of PTLD cells. We found that all the cell lines and cases of PTLD expressed SHP-1. Furthermore, PTLDs failed to express two proteins seen at the germinal center stage of B-cell differentiation: CD10 and BCL-6 (Carbone et al, 1998a; Cattoretti et al, 1995; Dent et al, 1997; Dogan et al, 2000; King et al, 2000). More than $40 \%$ of the cases expressed a post-germinal cell marker, CD138 (syndecan-1) (Bernfield et al, 1992; Sanderson et al, 1989). These data suggest that most, if not all, cases of B-cell PTLDs are derived from post-germinal center cells and that SHP-1 is an early marker of post-germinal center B-cell differentiation, more sensitive than CD138, which seems to be expressed mainly by the terminally differentiated immunoblasts and plasma cells.

\section{Results}

\section{PTLD-Related EBV ${ }^{+}$B Cells Express SHP-1 Protein In Vitro and In Vivo}

To determine whether cells closely resembling or derived from PTLDs express SHP-1, we analyzed by Western blotting three different $\mathrm{EBV}^{+}$lymphoblastoid $\mathrm{B}$-cell lines and one PTLD-derived $\mathrm{EBV}^{+} \mathrm{B}$-cell lymphoma cell line for expression of this protein. $T$ cellrich peripheral blood mononuclear cells (PBMC), known to express abundant SHP-1 (Zhang et al, $2000 b)$, served as a positive control. Two T-cell lines, PB-1 and 2B, which display diminished and nondetectable expression of SHP-1 (Zhang et al, 2000b), respectively, served as additional controls. As shown in Figure 1, all the lymphoblastoid B-cell lines $(\mathrm{A} 1, \mathrm{HH}$, and $\mathrm{RB}$ ) as well as the PTLD-derived lymphoma cell line (PTLD-1) expressed SHP-1 protein. The amount of SHP-1 expressed by these cells was comparable to that in PBMC and was much higher than in the PB-1 line, indicating high SHP-1 protein concentration in the PTLD-related EBV ${ }^{+}$B cells.

To determine whether SHP-1 protein is expressed by PTLD-related B cells in vivo, we examined three different xenotransplantation models using immunodeficient mice as recipients of human $\mathrm{EBV}^{+}$cells. In the first model (Majewski et al, 2000), the mice (three mice per cell line) were injected ip with four different $\mathrm{EBV}^{+}$Iymphoblastoid B-cell lines, identical or similar

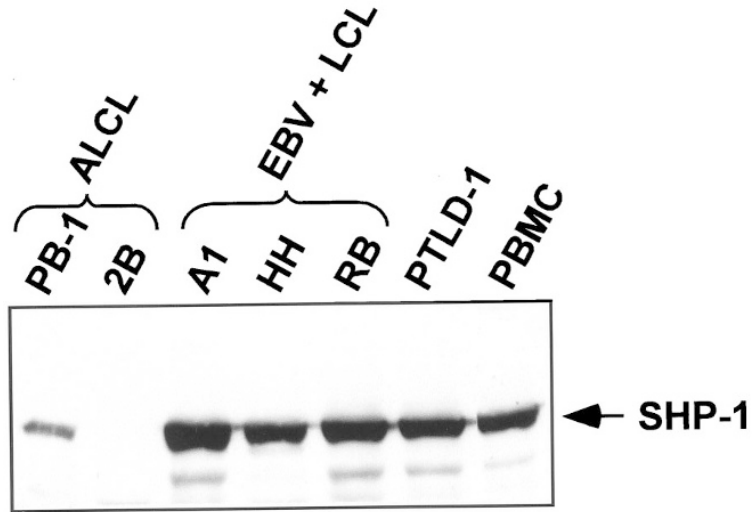

Figure 1.

Western blot analysis of SHP-1 protein expression by posttransplant lymphoproliferative disorder (PTLD)-related B-cell lines. Three EBV ${ }^{+}$lymphoblastoid B-cell lines $(A 1, H H$, and $R B)$ and a PTLD-derived EBV ${ }^{+}$B-cell lymphoma cell line (PTLD-1) were analyzed. Cutaneous T-cell lymphoma lines ( $P B-1$ and $2 B)$ that display diminished and absent expression of SHP-1 protein (Cattoretti, 1995) and normal PMBC, respectively, and that highly express SHP-1, served as controls.

to the ones used in the in vitro experiments (Fig. 1). In the second model, which was established to replicate even more closely PTLD development, PBMC from three healthy individuals were exposed briefly in vitro to EBV and then injected ip into the recipient mice (three to four mice per donor). All mice in both models developed ip tumors and, frequently, involvement of spleen, liver, and other internal organs within 4 to 6 weeks after the injection. In the third model, the PTLD-1 tumor was established directly from a pulmonary, $\mathrm{EBV}^{+}$, monoclonal PTLD, by subcutaneous implantation of the lymphoma tissue fragments (Majewski et al, 2001). The PTLD-1 tumor cells displayed the same characteristics as the original PTLD cells (Majewski et al, 2001). Identity of the tumor cells as being PTLD related was confirmed in all three models by histologic evaluation (a representative image of the tumor derived from the EBV-exposed normal PBMC is shown in Fig. 2A), detection of B-cell marker CD79a using human-specific antibodies (Fig. 2B), and expression of the EBV-encoded EBER-1 antigen (Fig. 2C). Immunohistochemical analysis of the primary tumors from all three PTLD models and, when applicable, of the involved internal organs, revealed strong SHP-1 protein expression in all the tissue sections examined (Fig. 2D depicts a representative staining). Thus, the above results for the cultured cells indicate that SHP-1 is strongly and universally expressed by the PTLD-related $\mathrm{EBV}^{+} \mathrm{B}$ cells both in vitro and in vivo.

\section{SHP-1 Expression in PTLD Tissue Samples}

Next, we sought to examine the pattern of SHP-1 expression directly in PTLD tissues. We evaluated a total of 58 cases of PTLD (Table 1) representing various morphologies, ranging from polymorphic hyperplasias to overt lymphomas with polymorphic (Fig. 

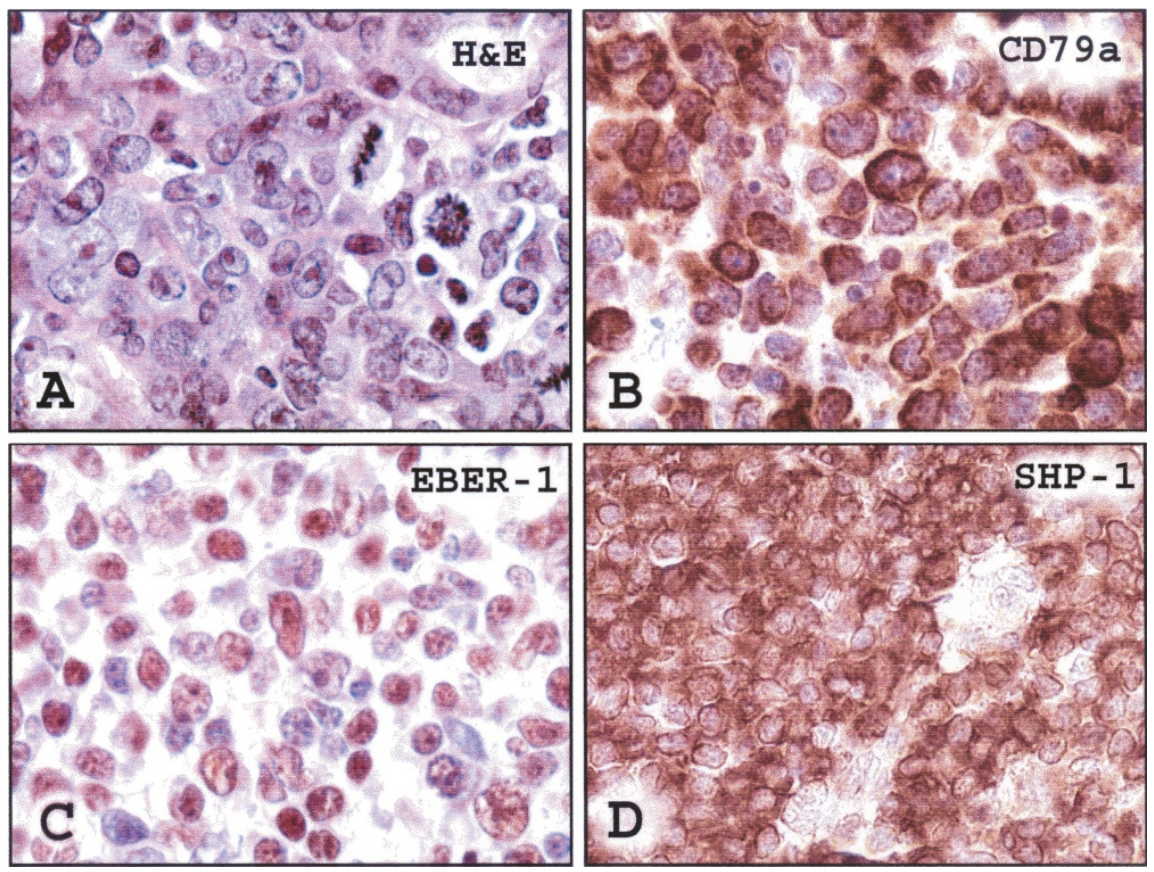

Figure 2.

Features of SHP-1 protein expression by the PTLD-related EBV ${ }^{+}$B-cell lymphoma. The lymphoma was derived from EBV-infected normal human peripheral blood mononuclear cells (PBMC) injected into immunodeficient mice. A, Hematoxylin eosin (HE) stain showing large-cell lymphoma with high mitotic rate. B, Immunoperoxidase stain with antibody against human CD79a (B-cell antigen) showing cell membrane staining in all lymphoma cells. C, In situ hybridization for EBV-encoded EBER-1 RNA showing nuclear staining in the lymphoma cells. D, Immunoperoxidase stain with an antibody against SHP-1 showing cytoplasmic staining in the lymphoma cells. These results are representative for all three types of EBV ${ }^{+}$PTLD-related tumors examined.

Table 1. Histologic Classification, Clonality, EBV Status, and CD138 Expression of PTLDs

\begin{tabular}{|c|c|c|c|c|c|c|c|}
\hline \multirow[b]{2}{*}{ Type } & \multirow[b]{2}{*}{ Case no. } & \multicolumn{3}{|c|}{ EBV status $^{a}$} & \multicolumn{3}{|c|}{ CD138 expression } \\
\hline & & + & - & $\mathrm{ND}^{b}$ & CD138+ & CD138- & $\mathrm{ND}^{b}$ \\
\hline Monomorphic & 28 & 21 & 6 & 1 & 9 & 17 & 2 \\
\hline Monoclonal $^{c}$ & 13 & 10 & 3 & & 1 & 12 & \\
\hline Polyclonal & 6 & 6 & & & 4 & 1 & 1 \\
\hline No expression & 5 & 3 & 2 & & 1 & 3 & 1 \\
\hline$N D^{b}$ & 4 & 2 & 1 & 1 & 3 & 1 & \\
\hline Polymorphic & 26 & 20 & 1 & 5 & 10 & 12 & 4 \\
\hline Monoclonal & 5 & 4 & 1 & & 2 & 3 & \\
\hline Polyclonal & 5 & 4 & & 1 & 3 & 1 & 1 \\
\hline No expression & 9 & 9 & & & 4 & 3 & 2 \\
\hline$N D^{b}$ & 7 & 3 & & 4 & 1 & 5 & 1 \\
\hline Others & 4 & & & & 4 & & 1 \\
\hline Multiple myeloma (all monoclonal) & 3 & 2 & 1 & & 3 & & \\
\hline Hodgkin's lymphoma (polyclonal) & 1 & 1 & & & & & 1 \\
\hline
\end{tabular}

${ }^{a}$ Determined by EBER-1 and/or LMP-1.

${ }^{b}$ Not determined.

${ }^{c}$ Clonality and immunoglobulin expression were determined by immunophenotyping.

$3 \mathrm{~A}$; representative case) and monomorphic morphology, frequently with immunoblastic and plasmacytoid differentiation (Fig. 3D; representative case). The cases were analyzed by immunohistochemistry using PTLD tissue sections prepared conventionally ( 37 cases) and as microarrays (21 cases with two to four 1.6-mm punch sections per case). In all examined PTLD cases, SHP-1 consistently showed strong immunoreactivity in at least $50 \%$ of the malignantappearing large lymphoid cells (Fig. 3, B and E, depicts the representative staining). In most cases, SHP-1 reactivity was identified in the vast majority of the PTLD cells.

Because of the universal expression of SHP-1 protein, we next evaluated the PTLD cases for expression of EBV to determine whether the presence of this virus was also universal in our case series. The EBV status as examined by EBER-1 and/or latent membrane protein-1 (LMP-1) expression was determined in 52 PTLDs (90\% of the cases); the results are summarized 


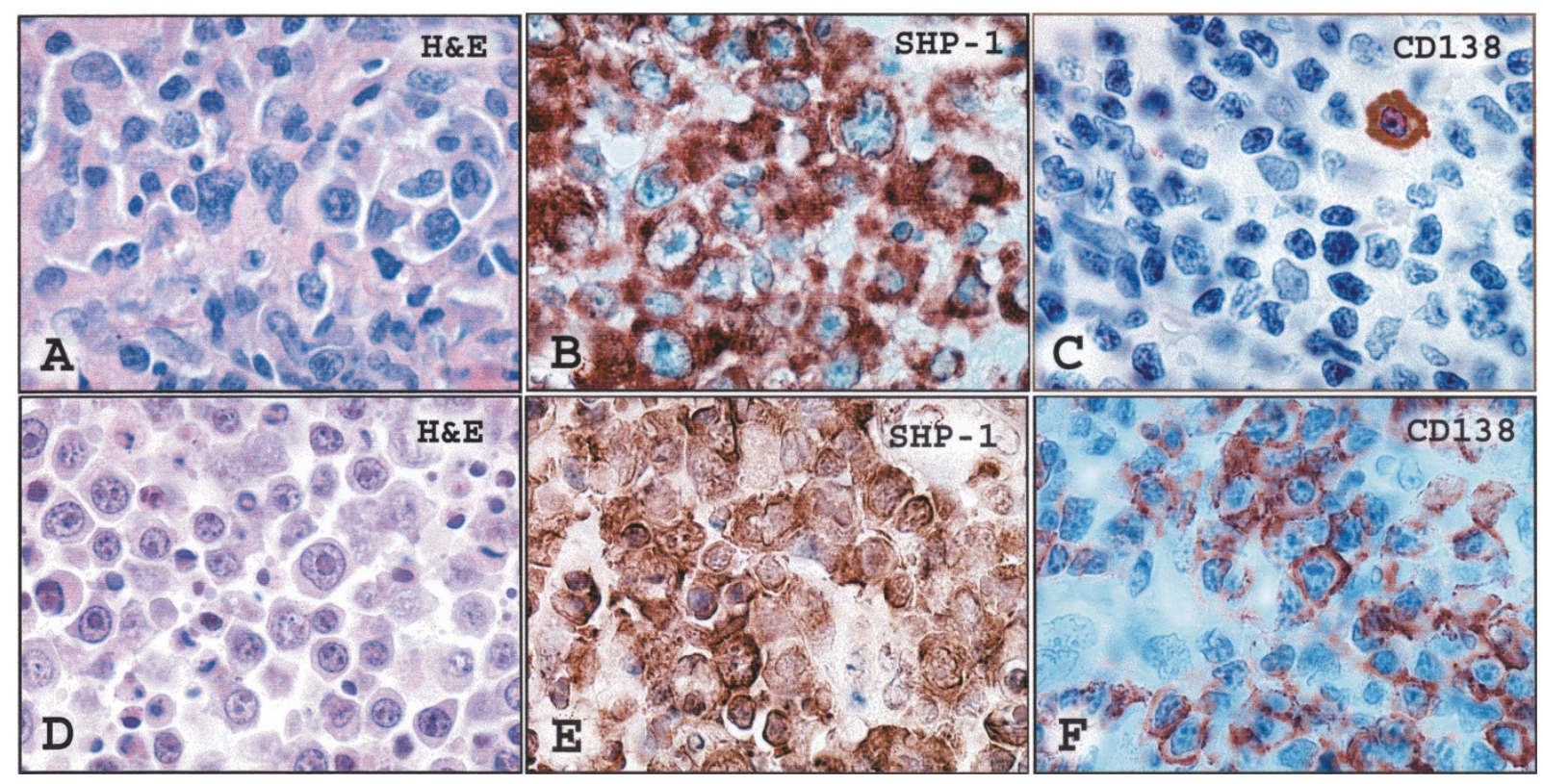

Figure 3.

Histology and expression of SHP-1 and CD138 by representative PTLD cases. A, HE stains of PTLD, polymorphic type. B, Immunohistochemical staining for SHP-1 protein showing strong staining of the malignant-appearing cells. C, Lack of staining of the lymphoma cells for CD138 protein (the depicted plasma cell represents an internal positive control). D, HE of PTLD, monomorphic type with immunoblastic and plasmacytoid differentiation. E, SHP-1 stains showing that the lymphoma cells are strongly positive for the protein. F, Strong CD138 staining in the immunoblasts and plasmacytic cells.

in Table 1. Although $44(85 \%)$ of these cases were $\mathrm{EBV}^{+}$, the remaining $8(15 \%)$ were $\mathrm{EBV}^{-}$. We did not find any qualitative difference in SHP-1 staining between the $\mathrm{EBV}+$ and $\mathrm{EBV}^{-}$cases; the percentage of positive cells and the intensity of cytoplasmic staining were comparable in both groups. These findings indicate that EBV infection does not seem to be required for SHP-1 expression and that other factors may be responsible for SHP-1 expression in the $\mathrm{EBV}^{-}$cases of B-cell PTLD.

\section{Expression in PTLD Specimens of Germinal Center B Cell (CD10 and BCL-6)-Associated and Post-Germinal Center B Cell (CD138)-Associated Proteins}

Because the universally positive SHP-1 staining suggested that most, if not all, PTLD cells represent a post-germinal center cell stage of B-cell differentiation, we analyzed by immunohistochemistry the expression of other proteins known to be differentially expressed by germinal and post-germinal center cells. The result was considered positive when at least $20 \%$ of the atypical and/or large cells stained. All 51 PTLD cases evaluated for CD10, a germinal center cell marker (Dogan et al, 2000; King et al, 2000), were negative for expression of this protein. Forty-two cases were stained for BCL-6, which is also restricted to the germinal center stage of differentiation (Carbone et al, 1998a, 1998b; Cattoretti et al, 1995; Dent et al, 1997; Dogan et al, 2000; King et al, 2000; Ye et al, 1997). Forty-one of these cases were negative for BCL-6 staining. In the one positive case, florid BCL-6 immunoreactivity was detected, with greater than $70 \%$ of the lymphoid cells staining. In this case, the tumor was classified morphologically as a polymorphic PTLD and was composed of predominantly intermediate-sized lymphocytes with scattered immunoblasts.

Next we examined 52 PTLD cases for expression of CD138, a member of the syndecan proteoglycan family, which is involved in cell-to-extracellular matrix interaction and is expressed by mature post-germinal center B cells, including immunoblasts and plasma cells, but not by germinal center cells (Bernfield et al, 1992; Sanderson et al, 1989). Expression of CD138 was not seen in 30 cases (Fig. 3C) but was detected in the remaining 22 cases, representing $42 \%$ of the cohort (Fig. 3F). The CD138 ${ }^{+}$cases encompassed diverse morphologic types of PTLD, with no particular type dominating (Table 1). The staining did, however, strongly correlate with the presence of overt immunoblasts and plasma cells, indicating that CD138 expression is confined to the $B$ cells representing terminal differentiation.

\section{Discussion}

The purpose of this study was to investigate the expression of SHP-1 in the whole spectrum of cell populations resembling or representing B-cell PTLDs to better define the histogenetic origin of these lymphoproliferative disorders. Recently we (Kossev et al, 2001) and others (Delibrias et al, 1997) have shown that SHP-1 expression is tightly regulated at specific stages of normal B-cell maturation. Furthermore, we found that the pattern of SHP-1 expression in small $B$-cell lymphomas reflects the maturation stage of their normal cell counterparts (Kossev et al, 2001). Because SHP-1 is expressed by post-germinal center, but not germinal center cells (Delibrias et al, 1997; 
Kossev et al, 2001), we attempted to identify the specific stage or stages of B-cell differentiation from which PTLDs are derived by evaluating SHP-1 expression in the PTLD cells.

PTLDs range from hyperplasias to aggressive lymphomas and are highly associated with EBV infection (Harris et al, 1997; Swierdlow, 1997). Our examination of the PTLD-like EBV ${ }^{+}$Iymphoblastoid B-cell lines, and the $\mathrm{EBV}^{+} \mathrm{B}$-cell lymphoma line derived directly from the PTLD tissue, demonstrated strong SHP-1 protein expression in all lines. Furthermore, the lymphoblastoid cell lines, the PTLD-derived lymphoma line, and the EBV-infected normal PBMC xenotransplanted into immunodeficient mice all gave raise to lymphoid tumors, which invariably expressed SHP-1 protein. We also examined SHP-1 expression in tissues from 58 cases representing a spectrum of PTLDs. All cases demonstrated strong SHP-1 immunoreactivity, including not only the $\mathrm{EBV}^{+}$but also the $\mathrm{EBV}^{-}$cases, suggesting that B-cell PTLDs correspond to post-germinal center $B$ cells regardless of their EBV infection status. We confirmed the postgerminal center origin of PTLD cells by demonstrating their lack of expression of germinal center cell markers CD10 and BCL-6 (with an exception of one BCL- ${ }^{+}$ case) and the presence of a post-germinal center cell marker CD138 in more than $40 \%$ of the cases. Based on these observations, we defined two major phenotypes in PTLDs, CD10 ${ }^{-} / \mathrm{BCL}_{-} 6^{-} / \mathrm{SHP}_{-} 1^{+} / \mathrm{CD} 138^{-}$ and $\mathrm{CD}^{-} 0^{-} / \mathrm{BCL}_{-} 6^{-} / \mathrm{SHP}-1^{+} / \mathrm{CD} 138^{+}$.

Previous studies investigating the phenotypic profiles in normal lymph nodes have shown that the precise stage of B-cell differentiation can be identified by analysis of the expression pattern of specific biologic markers, which are characteristic for the given stages of B-cell maturation (Carbone et al, 1998a, 1998b, 1999, 2001; Delibrias et al, 1997; Kossev et al, 2001). The phenotype BCL- $6^{+} / \mathrm{CD} 10^{+} / \mathrm{CD} 138^{-}$and the lack of SHP-1 expression correspond to germinal center B cells, whereas the phenotype $\mathrm{BCL}_{-}{ }^{-}$/ $\mathrm{CD}^{-} 0^{-} \mathrm{CD}_{138^{+}}$and the presence of SHP-1 expression correspond to post-germinal center B-cells. We have noted in normal reactive lymph nodes that many transformed, interfollicular, apparently post-germinal center B cells lack CD138 expression, whereas CD138 is expressed in the more terminally differentiated immunoblastic and plasmacytic B cells (M Paessler and MA Wasik, unpublished data). Accordingly, a recent study has described the early post-germinal center stage phenotype in which B cells are already BCL-6 ${ }^{-}$ but do not yet express CD138 (Carbone et al, 2001). Therefore, the CD10 ${ }^{-} / \mathrm{BCL}_{-} 6^{-} / \mathrm{SHP}_{-} 1^{+} / \mathrm{CD} 138^{+}$phenotype of PTLDs seems to correspond to the postgerminal center B cells at terminal stages of B-cell differentiation. In correlation with this phenotype, these PTLD cases tend to have an immunoblastic and plasmacytic cell morphology. The second phenotype, CD10 ${ }^{-} / \mathrm{BCL}-6^{-} / \mathrm{SHP}-1^{+} / \mathrm{CD}_{138^{-}}$, seems to represent an earlier stage of post-germinal center differentiation in which CD10 and BCL-6 are down-regulated and SHP-1 expression is up-regulated; however, the cells have not yet differentiated enough to express
CD138 (a schematic diagram of this concept is depicted in Fig. 4). The noted dichotomy of CD138 expression indicates that SHP-1 is a more sensitive marker of post-germinal B-cell differentiation than CD138 in both normal and PTLD cells. Because, as we have found (Kossev et al, 2001), SHP-1 is expressed not only on the post-germinal center cells but also on the pre-germinal center cells, one could argue that we did not exclude completely the possibility that some CD138- PTLD cases could be derived from pregerminal center B cells. However, the morphologic findings of the atypical lymphoid cells in the PTLDs, including large noncleaved cells with frequent immunoblastic features, are compatible with a stimulated postgerminal center stage of differentiation that has undergone immunoglobulin class switching, usually of the IgG immunoglobulin isotype. This histologic finding, coupled with the well-recognized role that EBV plays in driving B-cell maturation to the post-germinal center stage, as discussed in the next paragraph, argues against the possibility that these cells are unstimulated resting pre-germinal center cells. Structural analysis of the immunoglobulin sequence for evidence of somatic hypermutations in the PTLD cases in which clonal B cells have been identified could be considered to provide additional evidence for their post-germinal center cell derivation, particularly in the $\mathrm{EBV}^{-}$cases.

EBV seems, indeed, to play a major role in induction of the post-germinal center cell phenotype. Previous studies have shown that LMP-1, which is one of the key proteins responsible for the EBV-mediated cell transformation (Tanner and Alfieri, 2001; Tao and Wasik, 2001), is able to down-regulate expression of BCL-6 in germinal center B cells and drive their maturation to the post-germinal center stage (Cattoretti et al, 1997; Contreras-Broding et al, 1991; Rowe et al, 1994; Sample et al, 1991; Schaefer et al, 1991). Accordingly, the post-germinal center cell phenotype is not unique to the $\mathrm{EBV}^{+} \mathrm{B}$-cell PTLDs but is also present in the $\mathrm{EBV}^{+}$AIDS-related non-Hodgkin's lymphomas (Carbone et al, 1998a). In contrast, the EBV cases of such lymphomas are usually derived from

SHP-1+

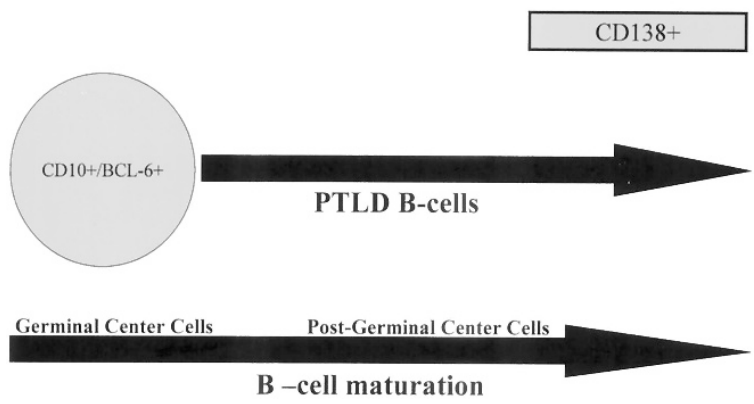

Figure 4.

Schematic diagram of PTLD cell derivation. Lack of expression of germinal center cell markers CD10 and BCL-6 (with the exception of a single case), universal expression of SHP-1, and expression of the CD138 by a subset of cases indicate that PTLD cells correspond to the normal post-germinal center B cells, either early (CD138-) or late (CD138+). 
germinal center stage B cells (Carbone et al, 1998a). Because our $\mathrm{EBV}^{-}$PTLD cases are invariably postgerminal in origin as determined by the SHP-1 expression, it seems that the pathogenesis of the $\mathrm{EBV}^{-}$ PTLDs and at least some $\mathrm{EBV}^{-}$AIDS-related lymphomas may be different. The two distinct immunophenotypes we identified in PTLDs showed no correlation with the EBV status. In fact, expression of not only EBER-1 but also of LMP-1 was seen in both CD138 ${ }^{+}$ and $\mathrm{CD}_{138^{-}}$cases. This finding suggests that the early post-germinal center phenotype is compatible with the LMP-1 expression, which may not always promote B-cell differentiation toward the late, CD138 ${ }^{+}$ stages.

The observation that one of our PTLD cases displayed florid BCL-6 reactivity is puzzling. Although BCL-6 is exclusively expressed at the germinal center cell stage of differentiation of normal mature B cells (Carbone et al, 1998a; Cattoretti et al, 1995; Dent et al, 1997; Ye et al, 1997), the strong SHP-1 staining in this case suggests a post-germinal cell origin. Furthermore, CD10 expression, which is a reliable marker of germinal center B cells (Dogan et al, 2000; King et al, 2000), is negative in this case, further arguing against germinal center cell derivation. We believe that the case represents either an early post-germinal center cell phenotype with an aberrant preservation of the BCL- 6 expression or, less likely, a late germinal center cell, or a transition stage, with an apparently premature strong expression of SHP-1. Interestingly, this case was negative for EBV. The lack of EBV-mediated down-regulation of BCL-6 expression and promotion of further B-cell maturation (Cattoretti et al, 1997; Contreras-Broding et al, 1991; Rowe et al, 1994; Sample et al, 1991; Schaefer et al, 1991) could have facilitated persistence of the BCL- 6 expression in this case.

In summary, assessment of the SHP-1 expression indicates that B-cell PTLDs display a post-germinal stage of differentiation regardless of the EBV infection status. Further analysis has identified two subsets of the PTLDs that differ in expression of CD138. The first subset, SHP $-1^{+} / \mathrm{CD} 10^{-} / \mathrm{BCL}-6^{-} / \mathrm{CD} 138^{+}$, seen in nearly half of the cases, reflects apparently late post-germinal center $B$ cells, whereas the second subset, SHP $-1^{+} / \mathrm{CD}^{-}{ }^{-}$/ BCL- $6^{-} / \mathrm{CD} 138^{-}$, corresponds to an early post-germinal center cell stage of B-cell differentiation. By virtue of being expressed earlier, SHP-1 is a more sensitive marker in identifying post-germinal B cells than CD138, which is expressed in the terminally differentiated immunoblastic and plasmacytic cells.

\section{Materials and Methods}

\section{PTLD-Related EBV ${ }^{+}$B-Cell Populations Cultured In Vitro and In Vivo}

Five PTLD-like lymphoblastoid B-cell lines (A1, HH, RB, $15 \mathrm{~A}$, and $20 \mathrm{~A}$ ) were established by in vitro infection with EBV of PBMC from three healthy individuals $(\mathrm{A} 1, \mathrm{HH}$, and $\mathrm{RB}$ ) and from two patients with low-grade small-cell lymphomas (15A and 20A) as described previously (Majewski et al, 2000). To establish an in vivo model of PTLD, four of the lines (A1, RB1, 15A, and 20A) were injected intraperitoneally into etoposide-pretreated, immunodeficient SCID mice (three mice per line) as described (Majewski et al, 2000). Most of the mice developed tumors within a 5-week period, involving peritoneum and frequently other organs such as liver, spleen, and kidneys. To establish a model that closely resembled the in vivo development of PTLD, PBMC from three healthy individuals were exposed for 45 minutes to the EBVcontaining supernatant of the marmoset B95/8 cell line and injected intraperitoneally at 7 to $10 \times 10^{6}$ cells per mouse into the etoposide-pretreated immunodeficient mice. All 11 mice (three to four per donor) developed intraperitoneal tumors within 4 to 6 weeks after the injection. The tumors and internal organs were harvested and analyzed by light microscopy. Representative sections were further evaluated by immunohistochemistry for expression of human B-cell markers (CD20 and CD79a) and EBV-related EBER-1, to confirm the origin and PTLD-like nature of the established tumors.

The PTLD-1 cell line was obtained from a liver transplant patient with a pulmonary, $\mathrm{EBV}^{+}$, monoclonal, polymorphic B-cell PTLD by subcutaneous implantation of the PTLD tissue fragments into an immunodeficient mouse (Majewski et al, 2001). Some of the tumor fragments harvested from the recipient mouse were propagated further in vitro as a cell suspension. The PTLD-1 cell line closely resembled the original tumor with regard to morphology, EBER-1 expression, and immunophenotype, including immunoglobulin lambda-light chain restriction (Majewski et al, 2001).

The EBV ${ }^{-}$T-cell lines PB-1 and 2B, used as controls in the in vitro studies, were established from a patient with T-cell lymphoma involving skin. The PB-1 cell line was obtained at a relatively early stage of the patient's cutaneous T-cell lymphoma, whereas the $2 \mathrm{~B}$ cell line was established at a later, aggressive stage of the disease (Zhang et al, 1996, 2000a, 2000b). In vitro cultures of the cell lines were performed at $37^{\circ} \mathrm{C}$ with $5 \% \mathrm{CO}_{2}$ in standard RPMI 1640 medium supplemented with $10 \%$ heat-inactivated fetal bovine serum, $1 \%$ penicillin/streptomycin/Fungizone mixture, and 2 $\mathrm{mmol} / \mathrm{L}$ L-glutamine (Zhang et al, 1996, 2000b). Control normal PBMC were obtained from healthy donors by a Ficoll-Paque purification (Zhang et al, 1996).

\section{PTLD Samples}

Fifty-eight cases of B-cell PTLD, diagnosed from patients who underwent solid organ transplantation, were identified in the files of the Department of Pathology and Laboratory Medicine at the Hospital of the University of Pennsylvania and Cleveland Clinic Medical Center. The corresponding formalin-fixed and/or B5-fixed paraffin-embedded tissue samples and clinical history files were retrieved. The paraffinembedded tissue samples in which the diagnosis of B-cell PTLD was rendered, represented heart, lung, kidney, liver, lymph node, and bone marrow biopsy samples from surgical and autopsy specimens. All cases were classified as PTLDs based on clinical history, morphology, immunohistochemistry, flow cy- 
tometry and, in most cases, EBV gene expression. The 58 cases studied were comprised of 26 cases of polymorphic B-cell PTLD, 28 cases of monomorphic B-cell PTLD, 3 cases of multiple myeloma/plasma cell dyscrasia, and 1 case of Hodgkin's lymphoma PTLD.

\section{Preparation of PTLD Tissue Microarray}

Twenty-one cases of PTLD from the Cleveland Clinic Medical Center were prepared as a tissue microarray. Representative areas of the PTLDs were cut on a tissue arrayer (Beecher Instruments, Silver Spring, Maryland) in 1.6- $\mathrm{mm}$ punch tissue sections and were sampled and arranged on a single paraffin tissue block. Two to four representative sections were sampled from each case and included into the microarray. The tissue microarray block and the conventional tissue blocks were serially sectioned and analyzed by light microscopy and immunohistochemistry.

\section{Western Blot Analysis}

Western blot analysis was performed as described previously (Zhang et al, 1996, 2000a, 2000b). In brief, the cells were washed and lysed in ice-cold lysis buffer. The lysates were separated on a $10 \%$ polyacrylamidesodium dodecyl sulfate gel and transferred electrophoretically onto hybridization transfer membranes. The membranes were blocked with $2 \%$ BSA in TBST buffer. To detect SHP-1 protein expression, the membranes were incubated with a SHP-1 polyclonal rabbit antibody (Santa Cruz Biotechnology, Santa Cruz, California) and later with the secondary goat anti-rabbit peroxidaseconjugated polyclonal antibody (Jackson ImmunoResearch Labs, West Grove, Pennsylvania). Blots were developed using ECL chemiluminescence reagents (Amersham Life Science, Arlington Heights, Illinois).

\section{Immunohistochemistry}

One representative tissue block was selected in each case for immunohistochemical staining. Formalin-fixed or B5-fixed, paraffin-embedded tissue was cut at 5- $\mu \mathrm{m}$ sections and placed on slides. The slides were stained with a monoclonal antibody to SHP-1 (Santa Cruz Biotechnology) using a standard avidin-biotin technique (Vector Laboratories, Burlingame, California) with antigen retrieval at $95^{\circ} \mathrm{C}$ for 20 minutes and, in a few cases, a catalyzed amplification method (CSA kit; DAKO, Carpinteria, California) (Kossev et al, 2001; Zhang et al, 2000b). In addition, the slides were stained with antibodies to CD10 (Novocastra Laboratories, Newcastle upon Tyne, United Kingdom), BCL-6 (Santa Cruz Biotechnology), and in case of the cultured cells, CD79 (DAKO), with antigen retrieval in Trilogy buffer (EDTA-based solution) using the avidin-biotin method and with an antibody to CD138 (Serotec, Oxford, United Kingdom) using the alkaline phosphatase anti-alkaline phosphatase method (Cordell et al, 1984).

\section{EBV Analysis}

Forty-nine (84\%) of the PTLD cases in this study and the PTLD-related xenotransplantation tumors har- vested from the recipient immunodeficient mice were analyzed for the presence of the EBV genome as described (Majewski et al, 2000) using in situ hybridization for EBER-1 with commercially available reagents (DAKO) and immunohistochemical staining with LMP-1-specific antibody (DAKO).

\section{Assessment of Immunohistochemical Staining}

All stained slides were examined independently by three members of our group and designated as positive or negative based on architecture and cytology. Examination of the cases included hematoxylin and eosin (HE) stains and routine B- and T-cell markers, SHP-1, CD10, BCL-6, and CD138. The rare cases that showed lack of convincing atypical cells in the immunohistochemically stained sections or ambiguous staining were not scored. SHP-1 immunoreactivity was considered positive if greater than $50 \%$ of the morphologically atypical lymphoid cells stained. Greater than $20 \%$ of the morphologically atypical lymphoid cells needed to show positive staining for CD10, BCL-6 (nuclear staining), and CD138 to be scored as positive. If present, epithelial cells served as positive internal controls for CD10. Reactive plasma cells served as positive internal controls for CD138.

\section{References}

Bernfield M, Kokenyesi R, Kato M, Spring J, Gallo RL, and Lose EF (1992). Biology of the syndecans: A family of transmembrane heparan sulfate proteoglycans. Annu Rev Cell Biol 8:365-393.

Carbone A, Gaidano G, Gloghini A, Larocca LM, Capello D, Canzonieri V, Antinori A, Tirelli U, Falini B, and Dalla-Favera R (1998a). Differential expression of BCL-6, CD138/ syndecan-1, and Epstein Barr virus-encoded latent membrane protein-1 identifies distinct histogenetic subsets of acquired immunodeficiency syndrome-related nonHodgkin's lymphomas. Blood 91:747-755.

Carbone A, Gloghini A, Gaidano G, Franceschi S, Capello D, Drexler HG, Falini B, and Dalla-Favera R (1998b). Expression status of BCL-6 and syndecan-1 identifies distinct histogenetic subtypes of Hodgkin's disease. Blood 92:2220-2228.

Carbone A, Gloghini A, Larocca LM, Antinori A, Falini B, Tirelli U, Dalla-Favera R, and Gaidano G (1999). Human immunodeficiency virus-associated Hodgkin's disease derives from post-germinal center B-cells. Blood 93:2319-2326.

Carbone A, Gloghini A, Larocca LM, Capello D, Pierconti F, Canzonieri V, Tirelli U, Dalla-Favera R, and Gaidano G (2001). Expression profile of MUM/IRF4, BCL-6, and CD138/ syndecan-1 defined novel histogenetic subsets of human immunodeficiency virus related lymphomas. Blood 98:744-751.

Cattoretti G, Chang CC, Cechova K, Zhang J, Ye BH, Falini B, Louie DC, Offit K, Chaganti RS, and Dalla-Favera R (1995). $\mathrm{BCL}-6$ protein is expressed in germinal-center B-cells. Blood 86:45-53.

Cattoretti G, Zhang J, Cleary AM, Lederman S, Gaidano G, Carbone A, and Chaganti RSK (1997). Downregulation of BCL-6 gene expression by CD40 and EBV latent membrane protein (LMP-1) and its block in lymphoma carrying Bcl-6 rearrangements. Blood 90:175a. 
Contreras-Broding BA, Anvert M, Imre S, Altiok E, Klein G, and Masucci MG (1991). B-cell phenotype dependent expression of the Epstein-Barr virus nuclear antigens EBNA1 mRNA and transcriptional initiation site. J Gen Virol 72:3025-3033.

Cordell JL, Falini B, Erber WN, Ghosh AK, Abdulaziz Z, MacDonald S, Pulford KA, Stein H, and Mason DY (1984). Immunoenzymatic labeling of monoclonal antibodies using immune complexes of alkaline phophatase and monoclonal antialkaline phosphatase (APAAP complexes). J Histochem Cytochem 32:219-229.

Delibrias CC, Floettmann JE, Rowe M, and Fearon DT (1997). Down-regulated expression of SHP-1 in Burkitt lymphomas and germinal center B-lymphocytes. J Exp Med 186:1575-1583.

Dent AL, Schaffer AL, Yu X, Allman D, and Staudt LM (1997). Control of inflammation, cytokine expression, and germinal center formation by BCL-6. Science 276:589-592.

Dogan A, Bagdi E, Munson P, and Isaacson PG (2000). CD10 and BCL-6 expression in paraffin sections of normal lymphoid tissue and B-cell lymphomas. Am J Surg Pathol 24:846-852.

Haque SJ, Harbor P, Tabrizi M, Yi T, and Williams BR (1998). Protein tyrosine phosphatase SHP-1 is a negative regulator of IL-4 and IL-13 dependent signal transductions. J Biol Chem 272:33893-33896.

Harris NL, Ferry JA, and Swierdlow SH (1997). Posttransplant lymphoproliferative disorders: Summary of Society for Hematopathology Workshop. Sem Diagn Pathol 14:8-14.

Harris NL, Swerdlow SH, Frizzera G, and Knowles DM (2001). Post-transplant lymphoproliferative disorders. In: Jaffe E, Harris NL, Stein H, Vardiman J, editors. World Health Organization Classification of Tumours: Tumours of the Hematopoietic and Lymphoid Tissues. Lyon: IARC Press, 264-271.

King BE, Chen C, Locker J, Kant J, Okuyama K, Falini B, and Swerdlow SH (2000). Immunophenotypic and genotypic markers of follicular center cell neoplasia in diffuse large B-cell lymphomas. Mod Pathol 13:1219-1231.

Kossev P, Raghunath PN, Schuster S, Tomaszewski J, Bagg A, and Wasik MA (2001). Differential expression of SHP-1 by malignant small B-lymphomas reflects the maturation stage of their normal B-cell counterparts. Am J Surg Pathol 25: 949-955.

Majewski M, Korecka M, Fields L, Kossev P, Schuler W, Shaw L, and Wasik MA (2001). Immunosuppressive signal transduction inhibitor RAD suppresses growth of posttransplant lymphoproliferative disorder (PTLD) cells. FASEB $J$ 15:A249.

Majewski M, Korecka M, Kossev P, Li S, Goldman J, Moore J, Silberstein LE, Nowell PC, Schuler W, Shaw LM, and Wasik MA (2000). Immunosuppressive macrolide SDZ RAD inhibits growth of human EBV-transformed B lymphocytes in vitro and in vivo: An approach to prevention and treatment of posttransplant lymphoproliferative disorders (PTLDs). Proc Natl Acad Sci USA 97:4285-4290.
Rowe M, Lear AL, Croom-Carter D, Davies AH, and Rickinson AB (1994). Three pathways of Epstein-Barr virus gene activation from EBNA-1 positive latency in B-lymphocytes. J Virol 66:122-126.

Sample J, Brooks L, Sample C, Young L, Rowe M, Gregory C, Rickinson A, and Kieff E (1991). Restricted Epstein-Barr virus protein expression in Burkitt lymphoma is reflected in a novel EBNA1 mRNA and transcriptional initiation site. Proc Natl Acad Sci USA 88:6343-6347.

Sanderson RD, Lalor P, and Bernfield M (1989). B lymphocytes express and lose syndecan-1 at specific stages of differentiation. Cell Regul 1:27-35.

Schaefer BC, Woisetschlager M, Strominger JL, and Speck SH (1991). Exclusive expression of Epstein-Barr virus nuclear antigen 1 in Burkitt's lymphoma arises from a third promoter distinct from the promoters utilized in latently infected lymphocytes. Proc Natl Acad Sci USA 88:6650-6554.

Swierdlow SH (1997). Classification of the posttransplant lymphoproliferative disorders: From the past to the present. Sem Diagn Pathol 14:2-7.

Tanner JE and Alfieri C (2001). The Epstein-Barr virus and post-transplant lymphoproliferative disease: Interplay of immunosuppression, EBV, and the immune system in disease pathogenesis. Transpl Infec Dis 3:60-69.

Tao $\mathrm{J}$ and Wasik MA (2001). Epstein-Barr virus associated polymorphic lymphoproliferative disorders occurring in nontransplant settings. Lab Invest 81:429-437.

Wu Y, Nadler MJ, Brennan LA, Gish GD, Timms JF, Fusaki N, Jongstra-Bilen J, Tada N, Pawson T, Wither J, Neel BG, and Hozumi N (1998). The B-cell transmembrane protein CD72 binds to and is an in vivo substrate of the protein tyrosine phosphatase SHP-1. Curr Biol 10:1009-1017.

Ye BH, Cattoretti G, Shen Q, Zhang T, Hawe N, deWaard R, Leung C, Nouri-Shirazi M, Orazi A, and Chaganti RS (1997). The BCL-6 proto-oncogene controls germinal center formation and Th2-type inflammation. Nat Genet 16:161-170.

Zhang Q, Nowak I, Vonderheid EC, Rook AH, Kadin ME, Nowell PC, Shaw LM, and Wasik MA (1996). Activation of the Jak-STAT signal transduction pathway associated with IL-2R common $\gamma$ chain in malignant T-cells derived from patients with cutaneous anaplastic large T-cell lymphoma and Seizure syndrome. Proc Natl Acad Sci USA 93:9148-9153.

Zhang J, Ally-Khan S, and Siminovitch KA (2000a). Roles of the SHP-1 tyrosine phosphatase in the negative regulation of cell signaling. Semin Immunol 12:361-378.

Zhang Q, Raghunath PN, Vonderheid E, Odum N, and Wasik MA (2000b). Lack of phosphotyrosine phophatase SHP-1 expression in malignant T-cell lymphoma cells results from methylation of the SHP-1 promoter. Am J Pathol 157:11371146. 